\title{
Parameter estimation and signal detection algorithm based on adaptive capture in non- cooperative communication
}

\author{
Zilong He, Peng Sun*, Kexian Gong, and Hua Jiang \\ Electronics and Communication Engineering, College of Information Engineering, Zhengzhou \\ University, 100 Science Avenue, Zhengzhou City, Henan Prov, China
}

\begin{abstract}
Aiming at the problem that the frequency offset in the noncooperative communication system causes the received signal spectrum to shift, which exceeds the passband of the matched filter and affects the subsequent demodulation, a parameter estimation and signal detection algorithm based on adaptive capture is proposed by this paper, which is more convenient for hardware implementation and consumes less resources. The algorithm is divided into three parts. Firstly, use the correlation value between the signal and the preamble sequence as the basis for frequency capture. Secondly, the frequency is accurately estimated based on the interpolation algorithm. Finally, the phase-locked loop structure is used to track the frequency according to the characteristics of the frequency gradually changing and the signal frequency offset is eliminated in the Digital Down Converter stage. It provides necessary conditions for accurate signal detection and phase estimation. The simulation results show that the algorithm has high estimation accuracy, wide esti-mation range and low complexity. It can also achieve better estimation accuracy and detection performance under low signal-to-noise ratio.
\end{abstract}

\section{Introduction}

In TDMA communication system, the signal transmission is dis-continuous and bursty which requires the receiver to accurately realize signal detection and fast synchronization. Especially in the non-cooperative communication system, because the carrier frequency is unknown, the signal will have a large frequency offset even up to the symbol rate of $20 \%$ above. The existence of frequency offset will cause the signal spectrum to shift. In the Digital Down Converter, the signal exceeds the passband of the matched filter, causing the baseband signal after Digital Down Converter to be incomplete and affecting the demodulation of subsequent signals. Therefore, it is necessary to eliminate the frequency offset before the digital down-conversion and ensure that the algorithm is con-venient for hardware implementation and consumes less resources.

Usually, a known leading sequence before the TDMA signal is used to estimate synchronize parameter.Therefore,for the demodulation of TDMA signals, the combination

\footnotetext{
*Corresponding author: iepengsun@zzu.edu.cn
} 
of open-loop estimation algorithm and closed-loop algorithm is usually adopted. The general TDMA signal demodulation process in satellite communication is shown in Figure 1:

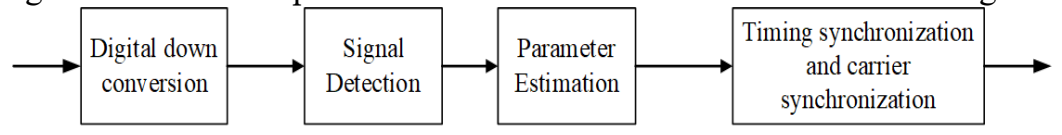

Fig.1. The general demodulation flow chart of TDMA signal.

Frequency estimation algorithms commonly used for TDMA signals are divided into two categories: frequency domain estimation algorithms and time domain esti-mation algorithms. The frequency domain estimation algorithm carries out a rough frequency estimation by searching the peak of the periodogram [1] and then uses an interpolation algorithm for accurate estimation [2-3]. Aiming at the large carrier frequency offset, liter-ature [4] proposes a large frequency offset estimation algorithm based on segmented FFT superposition, but it is not convenient for hardware imple-mentation. Time-domain estimation algorithms mainly use the autocorre-lation value of the eliminated modulation information signal to extract frequency offset information. This type of algorithm is generally more com-plex and the estimation range and estimation accuracy are mutually restr-icted [5-6]. Later, a time-domain improved algorithm based on correlation values was proposed which has low complexity and wide range [7]. But it has special requirements for the data frame structure.

The common existence detection algorithms for TDMA signals are di-vided into three categories: energy detection algorithm, frequency dete-ction algorithm and correlation detection algorithm. The basic principle of the energy detection algorithm is to use short-time energy as the detection feature. When the TDMA preamble sequence cannot be obtained, energy detection can be used as an applicable signal detection algorithm. The problem is that the algorithm is sensitive to noise and has poor detection performance under low SNR.

Frequency domain detection algorithms mainly include methods based on cyclic spectrum [8], methods based on amplitude spectrum [9] and DFT-based Power-Law algorithm [10]. This kind of algorithm can also achieve better performance under low SNR, but the implementation complexity is relatively high. The correlation detection algorithm is applicable to the situation where the leading sequence of data is known, but it is sensitive to frequency offset. So it is not applicable. In literature [11], the dual-correlation algorithm is proposed for detection. Although it can realize signal detection in the case of low SNR and frequency offset, the implementation complexity is relatively high.

In summary, the current general signal detection and parameter estimation algorithms cannot cope with the influence of frequency offset in non-cooperative communication on the signal and the parameter estimation and signal detection algorithms cannot balance complexity and accuracy. This paper proposes a burst signal parameter estimation and detection algorithm based on adaptive capture, which can eliminate the influence of frequency offset in the Digital Down Converter. The theoretical basis of the algorithm is given and a large number of simulations prove that the algorithm has better estimation and detection performance under low signal-to-noise ratio and large frequency offset.In addition, it consumes less resources.

The algorithm has the following advantages:

1) High accuracy and wide range of frequency estimation.

2) The hardware implementation consumes less resources.

3) It can eliminate the influence of frequency offset in non-cooperative communication on the signal and keep track of frequency changes. 


\section{Algorithm description}

\subsection{Principle of Digital Down Converter}

Digital Down Converter refers to the transfer of the effective spec-trum of the intermediate frequency signal after A/D sampling to the base-band through mixing, which can adjust the data rate at the same time. In general, Digital Down Converter consists of frequency conversion, filtering and resampling. In non-cooperative communication, there is frequency offset after Digital Down Converter since the frequency is unknown. If the frequency offset is large, the signal spectrum will be shifted so that the fil-ter module will affect the signal and the signal cannot be demodulated.

\subsection{Principle of adaptive capture algorithm}

Assuming that the transmission channel is an additive Gaussian white noise channel, the received signal model is as follows:

$$
r_{I F}(t)=\left[\sum_{k=-\infty}^{+\infty} a_{k} g_{T}(t-k T+\tau)\right] \exp \left[j\left(2 \pi\left(f_{c}+f^{\prime}\right)(t+\tau)+\theta_{0}\right)\right]+n(t)
$$

where $a_{k}$ is the modulation information symbol, $g_{T}(t)$ is the impulse response of the baseband shaping filter, $f_{c}$ is the IF frequency of the transmitter and $f^{\prime}$ is the difference between the local carrier frequency of the receiver and the actual frequency offset, $\theta_{0}$ is the phase offset, $n(t)$ is the complex Gaussian white noise. After sampling $r_{I F}(t)$ and Digital Down Converter, we get:

$$
r\left(n T_{s}\right)=\left[\sum_{k=-\infty}^{+\infty} a_{n} g_{T}\left(n T_{s}-k T+\tau\right)\right] \exp \left[j\left(2 \pi f^{\prime}(n+\tau) T_{s}+\theta_{0}\right)\right]+n\left(n T_{s}\right)
$$

In general, Root raised cosine function is used for forming filter in communication system. So we get:

$$
\sum_{k=-\infty}^{+\infty} g_{T}\left(n T_{s}-k T\right) \approx 1
$$

Finally, a simplified baseband signal expression is obtained:

$$
x_{k}=a_{k} e^{j\left(2 \pi f^{\prime}(k+\tau) T+\theta_{0}\right)}+n_{k}
$$

According to literature [12], the frequency estimation formula based on maximum likelihood is as follows:

$$
\hat{f}=\arg \max _{f}\left|\sum_{k=1}^{N} x_{k} a_{k}^{*} e^{-j\left(2 \pi f(k+\tau) T+\theta_{0}\right)}\right|
$$

It can be seen from formula (5) that when $f$ can maximize the above formula, $f$ is the maximum likelihood estimate of $f^{\prime}$. When $f$ takes $f^{\prime}$, expanding it to get $\left|a_{k}\right|^{2}$.

Without the complex number term, the accumulated value is the largest. Therefore, it can be proved that the correlation value between signal and leading sequence can be used as the basis for frequency capture. According to the principle of cross-correlation algorithm, the correlation calculation between the signal after Digital Down Converter signal and the known leading sequence can be obtained as follows:

$$
Y(i)=\sum_{k=1}^{N} x(k+i+\tau) a_{k}^{*}=\sum_{k=1}^{N}|a|^{2} e^{j\left(2 \pi \Delta f(k+i+\tau) T+\theta_{0}\right)}
$$


Let $\Delta f=f^{\prime}$, according to the frequency offset estimation formula derived from the maximum likelihood, when the frequency offset is $0,|Y(i)|$ takes the maximum value. It can be seen from Equation (1) that the actual frequency of the received signal is $f_{c}+f^{\prime}$ and the moving frequency of Digital Down Converter is $f_{c}$. Therefore, frequency offset can be captured through traversal and the moving frequency of Digital Down Converter can be changed step by step to approach the actual frequency. The frequency of one traversal is $f_{c}+n f_{b}$ or $f_{c}-n f_{b}$, where $f_{b}$ is the step value of one traversal. From the above derivation, we can see that $|Y(i)|$ takes the maximum value when $n f_{b}$ or ${ }^{-} n f_{b}$ is equal to $f^{\prime}$.

Since the magnitude and direction of the unknown frequency offset are random, it is necessary to further verify the specific influence of the frequency offset on the peak of the correlation result in order to ensure the frequency direction and accuracy. For making the correlation value more prominent, square the modulus of the correlation result to get:

$$
|Y(i)|^{2}=\left.\left.|| a\right|^{2} \sum_{k=1}^{N} e^{j 2 \pi \Delta f(k+i+\tau) T+\theta_{0}}\right|^{2}
$$

$i$ and $\tau$ are fixed values for the correlation value of a certain point. So expand $\sum_{k=1}^{N} e^{j 2 \pi \Delta f(k+i+\tau) T+\theta_{0}}$.

$$
\sum_{k=1}^{N} e^{j 2 \pi \Delta f(k+i+\tau) T+\theta_{0}}=\frac{1-\cos (-2 \pi \Delta f N)}{1-\cos (2 \pi \Delta f)}
$$

According to the even symmetry of the cosine function, we can get:

$$
|Y(i)|^{2}=A \frac{1-\cos (N 2 \pi \Delta f)}{1-\cos (2 \pi \Delta f)}=A \frac{\sin ^{2}(N 2 \pi \Delta f)}{\sin ^{2}(2 \pi \Delta f)}
$$

From Figure 2, we can intuitively see the specific impact of frequency offset on the correlation peak:

Combined with Figure 2, it can be seen that when the normalized frequency offset is within the range, the function is monotonic. As a whole, the peak value is larger when the frequency offset is closer to 0 . Therefore, it is further proved that the correlation result between the signal and the preamble sequence can be used as the basis for frequency capture. The following needs to determine the specific capture algorithm to ensure the accuracy and speed of frequency capture.

\subsection{Capture algorithm}

In actual communication, the frequency of burst signals is a gradual process but this change process is very slow. Therefor, we can assume that the frequency does not change during the frequency capture. Ideally, it is desirable to take the beginning of a burst as a traversal process and take the maximum value of the correlation result as the judgment basis. But in actual situations, the specific location and length of the burst cannot be obtained at this stage. Therefore, in order to ensure that every capture process contains burst signals. Performing a rough detection of the signal position through energy detection and then make a sliding correlation between the detected signal and the preamble sequence. Keep the maximum value of correlation results after one capture. In principle, it is necessary to ensure that every time the capture process contains at least one burst. 


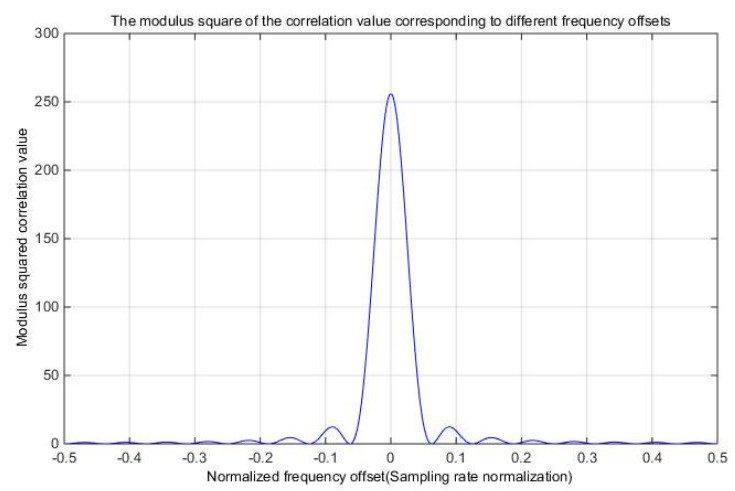

Fig. 2. The influence of frequency offset on correlation value.

\subsubsection{Capture model}

$$
\begin{gathered}
Y_{n}=|Y(i)|^{2}=\left.\left.|| a\right|^{2} \sum_{k=1}^{N} e^{j 2 \pi\left(f_{c}+f^{\prime}-\left(f_{c} \pm n f_{b}\right)\right)(k+i+\tau) T+\theta_{0}}\right|^{2} \\
f_{1}=f_{b} n_{\max }=f_{b} \arg \max _{n} Y(i)
\end{gathered}
$$

$f_{b}$ is the step value of the capture process. The ultimate goal is to search to make $n f_{b}=f^{\prime}$. For the sake of generality, normalize the energy of $Y_{n}$ and let $Y_{n}=\frac{|Y(i)|^{2}}{\sum_{k=1}^{N}|x(k+i)|^{2}}$.

\subsubsection{Rough estimate}

1) The threshold is set to $T h$ according to Figure 2 and the corresponding frequency is $f_{t h}$. In order to ensure that the capture performance threshold under low signal-to-noise ratio should be in an appropriate range, the threshold is set to $25 \%$ of the peak value through simulation and comparison. Traversing with the initial DDC frequency $f_{c}$ as the initial value which means $n=0$. By comparing the size of $Y_{0}$ and $T h$, it is judged whether the range of frequency offset is within or outside the threshold.

2) If $Y_{0}<T h$, prove the initial frequency offset value $\left|f^{\prime}>\right| f_{t h}||$. Therefore, set the step value at this time to $f_{b}=f_{\text {th }}$ and start traversing in both directions until $Y_{n}>T h$. At this time $f^{\prime}-f_{b} n<f_{\text {th }}$, it proves that the frequency offset at this time has been reduced to the threshold range. At the same time, the direction of the frequency offset can be determined according to the direction of the step value at this time. If $Y_{0}>T h$, prove the initial frequency offset value $\left|f^{\prime}<\right| f_{t h}||$. Although the size range of the frequency offset is determined at this time, the direction of the frequency offset is unknown. 
According to the above-mentioned monotonicity, we determine the frequency offset direction at this time by performing a search in two directions.

3) At this time, the correlation peak satisfies $Y_{n}>T h$. Since the direction of the frequency is already known, it is necessary to continue traversing in order to find the maximum peak. According to the monotonicity of the influence of frequency on the correlation peak, there must be $Y_{n}<T h$ stepping in a known direction. Stop capturing when $Y_{n}<T h$. We select the largest peak value as the frequency offset of the rough estimation stage. The rough estimate frequency value is $f_{1}=n f_{b}, f_{1}=n f_{b}$. In order to improve the capture speed of large frequency offsets and ensure the reliability of the correlation value, the $f_{\text {th }}$ is relatively large. This will result in low frequency resolution and insufficient accuracy of the captured frequency values, which requiring accurate estimation.

\subsubsection{Accurate estimate}

According to the previous analysis, the largest peak and its two adjacent peaks both contain frequency offset information. Therefore, the two peaks adjacent to the maximum peak can be used for interpolation to further accurately estimate the frequency offset. From equation (9), we can get:

$$
\begin{gathered}
\Omega(n)=|Y(i)|=\sqrt{A} \frac{\sin (N 2 \pi \Delta f)}{\sin (2 \pi \Delta f)} \\
\Delta f=\left(f^{\prime}-n f_{b}\right) / f_{s}, \text { let } \frac{f_{b}}{f_{s}}=\frac{1}{2 N}, \xi=\frac{f^{\prime}}{f_{b}} \text {. Finally get: } \\
\Omega(n)=\sqrt{A} \frac{\sin \left(\pi N\left(\frac{\xi f^{\prime}}{f_{s}}-n \frac{1}{2 N}\right)\right)}{\sin \left(\pi\left(\frac{\xi f^{\prime}}{f_{s}}-n \frac{1}{2 N}\right)\right)}=\sqrt{A} \frac{\sin \left(\frac{\pi}{2}(\xi-n)\right)}{\sin \left(\frac{\pi}{2 N}(\xi-n)\right)} \\
\hat{\xi}=\arg \max \left(Y_{n}\right)
\end{gathered}
$$

Use the interpolation formula in [13]:

$$
\Delta \hat{\xi}=\frac{\Omega(\hat{\xi}+1)-\Omega(\hat{\xi}-1)}{\Omega(\hat{\xi}+1)+\Omega(\hat{\xi}-1)}
$$

It is proved that $\Delta \hat{\xi}$ is an unbiased estimate of the remaining frequency offset. It should be noted that there are restrictions on the use of the above interpolation formula. It is needed to ensure that $f_{b}=\frac{f_{s}}{2 N}$. Therefore, set the step value of the third step of the rough estimation stage to $\frac{f_{s}}{2 N}$. The final frequency estimate is:

$$
f_{\text {fin }}=f_{1}+\Delta \hat{\xi}_{b}
$$




\subsubsection{Signal detection}

After the frequency is determined, set the Digital Down Converter frequency to $f_{c}+f_{\text {fin }}$. At this time, the signal after Digital Down Converter is the signal with frequency offset eliminated and the signal can be detected directly by using equation (9). In order to ensure that the thresholds of signals of different amplitudes are uniform during detection. It is necessary to normalize the signal energy. The signal detection decision expression based on the correlation algorithm is as follows:

$$
U(i)=\frac{|Y(i)|^{2}}{\sum_{k=1}^{N}|x(k+i+\tau)|^{2}}
$$

\subsubsection{Use correlation results to find phase offset}

The expression of data-assisted phase offset estimation in [14] is as follows:

$$
\hat{\varphi}=\arg \left\{\sum_{k=1}^{N} y_{k} a_{k}^{*}\right\}
$$

And

$$
y_{k}=x(k+i+\tau)=a_{k} e^{j 2 \pi\left(f^{\prime}-\hat{f}\right)(k+i+\tau) T+\theta_{0}}
$$

The correlation value after frequency capture and signal detection is as follows:

$$
Y(i)=\sum_{k=1}^{N}|a|^{2} e^{j\left(2 \pi\left(f^{\prime}-f_{f i n}\right)(k+i+\tau) T+\theta_{0}\right)}
$$

$f^{\prime}-f_{\text {fin }}$ is small enough to be ignored. Therefore, we can directly use the correlation value in the signal detection to estimate the phase offset and only need to perform the argument calculation on the correlation value. It does not need to be calculated separately, reducing the computational complexity. The phase offset estimation expression is as follows:

$$
\hat{\varphi}=\arg \{Y(i)\}
$$

\subsubsection{Frequency tracking}

In this paper, the frequency estimation algorithm based on adaptive capture needs several of times to capture the frequency and then perform accurate estimation. It is assumed that the frequency does not change during the capture process but the frequency will slowly change over time. Therefore, it needs to be able to track the frequency as the frequency changes after frequency capture in practical applications. Phase-locked loop as the core structure of closedloop carrier synchronization algorithm, its main function is to capture and track signal frequency [15]. Therefore, on the basis of the algorithm in this paper, the phase-locked loop structure is applied to the frequency tracking stage. A phase detection module, a loop filter and a numerically controlled oscillator are added after the signal detection. The numerically controlled oscillator is used to adjust the frequency of the Digital Down Converter module. In this way, a closed-loop structure is formed as a whole to realize the tracking of the slowly changing frequency.

The overall flow of the algorithm is shown in the figure below: 


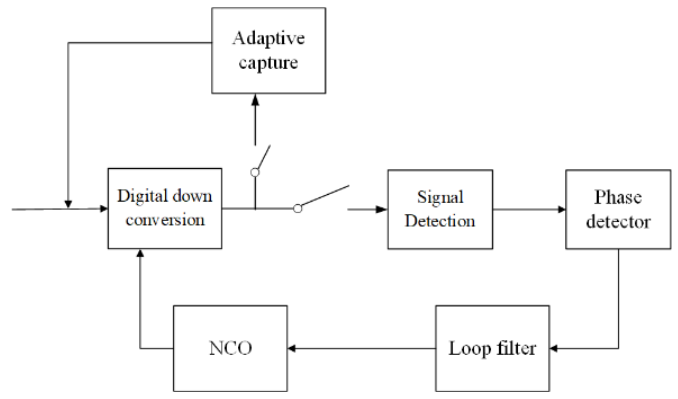

Fig. 3. Parameter estimation and burst detection process based on adaptive capture.

\section{Performance evaluation}

The simulation of the algorithm in this paper is mainly developed from two aspects: algorithm complexity and algorithm performance.

\subsection{Algorithm complexity}

The frequency estimation algorithm in this article is before signal detection. Although energy detection is used to find the beginning of the signal at this time, the position of the valid data is not accurate. Whether using the FFT-based frequency estimation algorithm or the adaptive frequency capture algorithm in this article, multiple sliding correlations are still needed to ensure the accurate correspondence between the preamble sequence and the valid data. In hardware implementation, computing modules can be reused. Therefore, we mainly compare the complexity of an operation when calculating complexity. We analyze the algorithm complexity from the perspective of hardware resource consumption.

Table 1. Frequency estimation algorithm based on FFT.

\begin{tabular}{|c|c|c|c|c|}
\hline Algorithm & $\begin{array}{c}\text { Multiplica- } \\
\text { tion }\end{array}$ & Add & division & $\begin{array}{c}\text { Square } \\
\text { root }\end{array}$ \\
\hline FFT & $\begin{array}{l}4 N \log _{2}(2 N) \\
+4 N\end{array}$ & $\begin{array}{c}N\left(6 \log _{2}(2 N)\right. \\
+4)+1+2\end{array}$ & 2 & 2 \\
\hline $\begin{array}{c}\text { Adaptive capture } \\
\text { algorithm }\end{array}$ & $4 N+4$ & $4 N+5$ & 2 & 2 \\
\hline
\end{tabular}

Table 2. Comparison of operational complexity of signal detection module.

\begin{tabular}{|c|c|c|c|c|}
\hline Algorithm & $\begin{array}{c}\text { Multiplica- } \\
\text { tion }\end{array}$ & Add & division & $\begin{array}{c}\text { Square } \\
\text { root }\end{array}$ \\
\hline $\begin{array}{c}\text { Double correlation } \\
\text { algorithm }\end{array}$ & $\begin{array}{c}\frac{N}{L}\left(4 N+2 \frac{N}{L}\right) \\
-4 N-2\end{array}$ & $\begin{array}{c}4 \frac{N^{2}}{L} \\
-4 N\end{array}$ & 1 & $\frac{N}{L}-1$ \\
\hline $\begin{array}{c}\text { Adaptive capture } \\
\text { algorithm }\end{array}$ & $4 N+4$ & $4 N+5$ & 2 & 2 \\
\hline
\end{tabular}


Table 3. Comparison of capture times.

\begin{tabular}{|c|c|c|c|}
\hline Algorithm & $5 \%$ & $10 \%$ & $20 \%$ \\
\hline $\begin{array}{c}\text { Algorithm based on interpolation } \\
\text { capture }\end{array}$ & 5 & 8 & 15 \\
\hline $\begin{array}{c}\text { Algorithm for conventional step } \\
\text { capture }\end{array}$ & 50 & 100 & 200 \\
\hline
\end{tabular}

According to the analysis from the perspective of resource consumption in Table 1 , the complexity of the adaptive capture algorithm saves nearly $80 \%$ compared to the FFT frequency estimation algorithm when $N=128$. But the adaptive capture algorithm requires more data to capture than the FFT frequency estimation algorithm, which brings about time consumption. Since time is only consumed in the initial capture stage, the impact is negligible.

Comparison of operational complexity of signal detection module:

When comparing the complexity with the signal detection algorithm against frequency offset in [11], the complexity of the frequency estimation module is taken as part of the calculation since the signal detection module of the adaptive acquisition algorithm performs signal detection after the frequency estimation module. Table 2 compares the operational complexity of the signal detection module.

Comparison of traversal times of different capture algorithms:

Compared with the conventional step capture algorithm, the algorithm based on interpolation capture proposed in this paper reduces the number of steps. Conventional capture refers to setting a more precise step value and traversing in two directions. Take the maximum value corresponding to the frequency as the final frequency estimate. Table 3 shows the comparison of the capture times of the two algorithms in different frequency offset ranges.

\subsection{Algorithm performance}

\section{Simulation 1: Accuracy and range of frequency capture}

The mean square error is used to measure the frequency estimation accuracy of the adaptive capture algorithm. The signal used in the algorithm is a single carrier signal modulated by QPSK.Set the normalized frequency offset to 0.02 and 0.1 (normalized symbol rate). The preamble length is $N=128$. The correlation function interval of $L \& R$ and Fitz algorithm is taken as $\frac{N}{2}$. The number of simulations under each signal-to-noise ratio is 10000 . The simulation result is shown in Figure 4 and Figure 5.

From the simulation results in Figure 4 and Figure 5, it can be seen that the frequency estimation mean square error of the adaptive capture algorithm and the $L \& R$ and Fitz algorithms in this paper can be approximated to $M C R B$ under the condition that the normalized frequency offset is 0.02 . The $L \& W$ algorithm is difficult to approach $M C R B$ even under high signal-to-noise ratio conditions. When the normalized frequency offset is 0.1 , the $L \& R$ and Fitz algorithms can no longer perform frequency estimation because the frequency offset exceeds the estimation range of the algorithm. Although the estimation range of the $L \& W$ algorithm is larger, its estimation accuracy is poor. The mean square error of frequency offset estimation of the adaptive capture algorithm in this paper can still be close to $M C R B$ under the condition that the normalized frequency offset is 0.1 . In theory, the 
frequency offset estimation range of the algorithm can reach $\pm 25 \%$ of the sampling rate under the condition of four times oversampling.

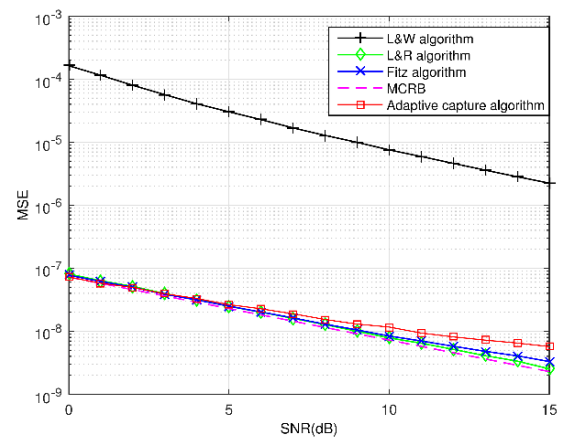

Fig. 4. Different frequency estimation algorithms estimate performance curve (Normalized frequency offset 0.02).

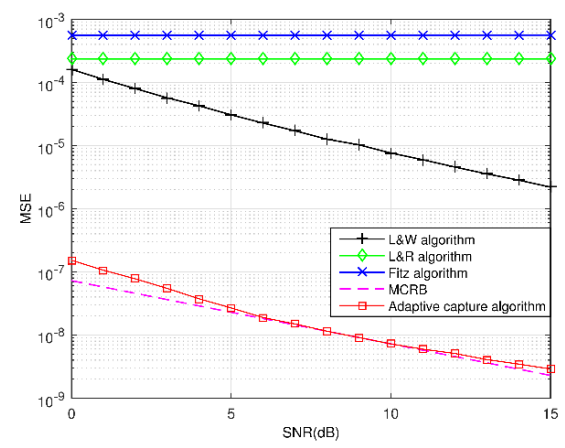

Fig. 5. Different frequency estimation algorithms estimate performance curve (Normalized frequency offset is 0.1 ).

\section{Simulation 2: The influence of pilot frequency on search accuracy}

In order to verify the influence of the pilot frequency on the estimation accuracy of each algorithm, set the pilot length $N=100$. The correlation function interval of $L \& R$ and Fitz algorithm is taken as $\frac{N}{2}$. The number of simulations under each signal-to-noise ratio is 10000 . Set the normalized frequency offset to 0.02 (normalized symbol rate) and get the simulation result shown in Figure 6.

From the comparison of the simulation results in Figure 5 with Figure 4, it can be seen that the adaptive capture algorithm in this paper and the $L \& R$ and Fitz algorithms still have the highest accuracy. The overall trend of each algorithm remains unchanged. But the overall accuracy of the algorithm decreases slightly. It can be seen that the increase of the pilot length can improve the estimation accuracy of the algorithm.

\section{Simulation 3: Signal detection probability}

Generate a burst signal whose modulation method is QPSK. The first 32 symbols are noise. Then a 32-symbol preamble sequence is added. After the data, it is 936 symbols of valid data and three consecutive segments of data in this format are used for signal detection performance simulation. The normalized frequency offset is set to 0.05 and the simulation is performed 10000 times under different signal-to-noise ratios. The detection probability and false alarm probability are counted. 
It can be seen from Table 4 that after the frequency offset is eliminated based on the adaptive capture algorithm, the corrlelation algorithm with better anti-noise performance can be used for burst signal detection and the detection performance still has a better effect at a lower signal-to-noise ratio.

Table 4. Signal detection probability.

\begin{tabular}{|c|c|c|c|c|c|}
\hline ber $\left(\frac{E_{b}}{N_{0}}\right)$ & -5 & -4 & -3 & -3 & -3 \\
\hline $\begin{array}{l}\text { Probability } \\
\text { of detection }\end{array}$ & $99.9 \%$ & $100 \%$ & $100 \%$ & $100 \%$ & $100 \%$ \\
\hline $\begin{array}{l}\text { False alarm } \\
\text { probability }\end{array}$ & $3.3639 * 10^{-6}$ & $3.0581 * 10^{-7}$ & $1.5291 * 10^{-7}$ & 0 & 0 \\
\hline
\end{tabular}

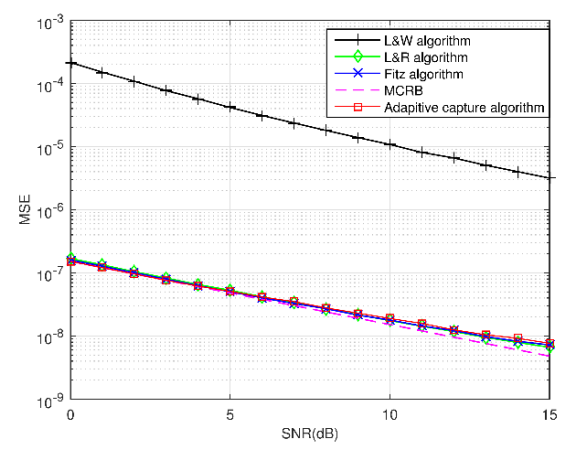

Fig. 6. The influence of pilot frequency on the accuracy of frequency offset estimation( $N=100$ and Normalized frequency offset is 0.02 ).

\section{Simulation 4: Phase offset estimation accuracy}

The mean square error is used to measure the phase offset estimation accuracy of this article. The signal used in the algorithm is a single carrier signal modulated by QPSK. In order to verify that the frequency estimation accuracy of the adaptive capture algorithm meets the requirements of phase offset estimation and the estimation performance of the phase offset estimation algorithm. We use different frequency offset estimation algorithms to eliminate the frequency offset data for phase offset estimation simulation. Set the normalized frequency offset to 0.02 and the length of the preamble $N$ to128. The correlation function interval of the $L \& R$ algorithm is $\frac{N}{2}$ and the simulation times are 10000 times under each SNR.

It can be seen from Figure 7 that using the adaptive capture frequency offset estimation or the $L \& R$ algorithm to eliminate the frequency offset and then using the intermediate results for phase offset estimation can achieve high accuracy. It is proved that the estimation accuracy of the adaptive capture frequency offset algorithm meets the requirements of phase offset estimation. The mean square error of the phase offset estimation algorithm is close to $M C R B$. 


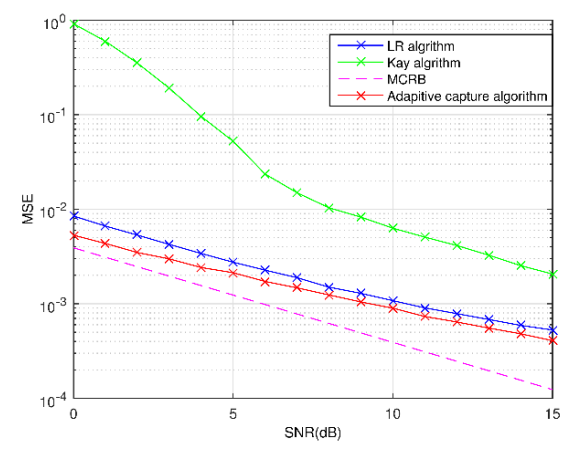

Fig. 7. Phase offset estimation performance curve $(N=128$ and Normalized frequency offset is $0.05)$.

\section{Conclusion}

This paper proposes a parameter estimation and signal detection algorithm based on adaptive capture in non-cooperative communication. The basic idea of the algorithm is to capture multiple times through sliding correlation and find the frequency corresponding to the maximum correlation value as the rough estimated frequency value.After derivation, an interpolation formula suitable for this algorithm is found for accurate estimation. On the basis of finding the frequency, the correlation result can be used for signal detection and phase offset estimation with simple calculations. Finally, the entire parameter estimation and signal detection module constitutes a closed-loop structure, which is based on the principle of phaselocked loop to realize the tracking of the slowly varying frequency. The algorithm eliminates the frequency offset in the Digital Down Converter stage to prevent the large frequency offset from affecting the signal.The simulation shows that the estimation accuracy of this algorithm is close to $M C R B$. The estimation range is wide and has better performance under low SNR. The hardware-based implementation consumes less resources.Although multiple captures are required to obtain the estimated value, it is only necessary to perform a complete frequency capture at the beginning. After obtaining an accurate estimate, the phase-locked structure can be used for frequency tracking. Therefore, the algorithm in this paper can be applied to the TDMA communication system.

This work was supported by the National Natural Science Foundation of China under Grant U1736107

\section{References}

1. F. Mazzenga and G.E.Corazza.Blind least-squares estimation of carrier phase, Doppler shift, and Doppler rate for m-PSK burst transmission. IEEE Communications Letters.2(3),73-75.(1998)

2. Dae-Ki Hong, Dong-Joo Kim, Young-Jo Lee, Suk-Hyon Yoon, Daesik Hong and Chang-Eon Kang. A simple interpolation technique for the DFT for joint system parameters estimation in burst MPSK transmissions. IEEE Transactions on Communications. 51(7),1051-1056.(2003)

3. Cui lian Wang; Yin Li; Ji yang Yu; Ke Li. Carrier synchronization high-precision FFT frequency offset estimation algorithm for deep-space communication. Spacecraft Engineering. 27(6), 63-68.(2018) 
4. Zhan Ye.Reception technique and reality of low SNR about satellite communication. PLA University of Science and Technology. (2010)

5. M Luise;R Reggiannini.Carrier frequency recovery in all-digital modems for burstmode transmission. IEEE Transactions on Communications.43(3),1169-1178.(1995)

6. Fitz, M.P.Planar filtered techniques for burst mode carrier synchronization. IEEE Global Telecommunications Conference.(1991)

7. Heng zhou Xu, Hai Zhu, Si feng Zhu, Zhong yang Yu.A wide range and low complexity carrier frequency offset estimation algorithm.Electronic Journal.47(12),2550-2555.(2019)

8. Xu Zhu, Takeo Fujii.Modulation Classification in Cognitive Radios for Satellite and Terrestrial Systems. IEEE International Conference on Communication Workshop.1612-1616.(2015)

9. WANG Q Y, XIE Z D, HU J.Blind Detection of Satellite Communication Signals Based on Cyclic Spectrum. 2015 International Conference on Wireless Communications and Signal Processing.7341216.(2015)

10. RIGLING B D.Maximum Likelihood Estimation of Band-limited Power Law Spectrums.19(5),307-310.(2012)

11. Zhang, P.Z.An improved algorithm for burst signal detection in TDMA system.Electronic technology and software engineering.13,40-41.(2016)

12. Mengali, Umberto and D'Andrea, Aldo N.Synchronization Techniques for Digital Receivers. Applications of Communications Theory. 10.1007/978-1-4899-1807-9.(1997)

13. Chao Gong, Bang ning Zhang, Dao xing Guo.FFT-based fast and high precision carrier parameter joint estimation algorithm.Electronic Journal.38(4),766-770.(2010)

14. P.Y.Kam.Maximum-likelihood carrier phase recovery for linear suppressed-carrier digital data modulations.IEEE Transactions on Communications.34(6),522-527.(1986)

15. Floyd M.Gardner.Phaselock techniques(Third Edition,Wiley,2005) 